\title{
Laser Direct-Write for Low AC Loss High Temperature Superconductor Applications
}

\author{
Kristin M. METKUS, Michael S. OSOFSKY, Nicholas A. CHARIPAR, and Alberto PIQUÉ \\ Naval Research Laboratory, Materials Science and Technology Division, Code 6364 \\ 4555 Overlook Ave. SW, Washington, DC 20375, USA \\ E-mail: kristin.metkus@nrl.navy.mil
}

\begin{abstract}
The use of high temperature superconductors (HTS) in applications such as power cables, fault current limiters, and electromagnets, has seen rapid growth in recent years. While HTS exhibit zero dc resistance, appreciable ac losses are observed when the HTS are subject to an applied ac magnetic field. By dividing the HTS films into thin filament arrays and creating periodic metallic cross-overs to allow for flux penetration, the ac loss of the HTS can be significantly reduced. The HTS, $\mathrm{YBa}_{2} \mathrm{Cu}_{3} \mathrm{O}_{7-x}$ (YBCO), films were prepared from the metal organic decomposition of a trifluoroacetate-based (TFA) precursor solution onto buffered substrates, manufactured by AMSC. To deposit the TFA solutions onto the buffered substrates, a new laser direct-write (LDW) technique was developed. LDW is a non-contact, non-lithographic process that relies on the mask-less printing of functional materials, such as metallic inks. The transferred TFA precursor filaments were then decomposed and subsequently characterized. In addition to the deposition of TFA precursors onto buffered substrates, LDW was used to print silver lines for metallic crossovers onto HTS tapes. These results show LDW to be a promising technique for the fabrication and modification of high temperature superconductors.

DOI:10.2961/jlmn.2013.01.0005
\end{abstract}

Keywords: high temperature superconductor (HTS), metal organic decomposition, YBCO, laser direct-write, rapid prototyping

\section{Introduction}

Recently, high temperature superconductors (HTS) have emerged as a viable technology for many applications, including power distribution, motors, and magnets, to name a few. The zero dc resistance of HTS enables them to carry large currents at ambient magnetic fields and thus enables a reduction of conductor weight and volume of various systems. The development of $2^{\text {nd }}$ generation conductors, which consist of flat metal tapes coated with thin oxide buffer layers and a thin $\mathrm{YBa}_{2} \mathrm{Cu}_{3} \mathrm{O}_{7-x}$ (YBCO) HTS coating, has provided a platform for many applications because of the ability to produce long lengths of material (many kilometers). [1] However, the flat architecture of these tapes causes significant losses for ac applications, where the tapes are exposed to ac magnetic fields. While it is known that ac losses can be greatly reduced by using a twisted wire geometry, the flat tape architecture prevents this formation.

It is well established that ac losses can be significantly reduced in HTS tapes by dividing the YBCO film into narrow filament arrays (Figure 1). Losses can be further minimized by the periodic introduction of filament crossover bridges along the conductor length that allow for flux penetration between all of the filaments. [2-4] This simulated twisted pair geometry on the flat tapes, seen in Figure 2, offers a solution to reduce the induced ac losses.

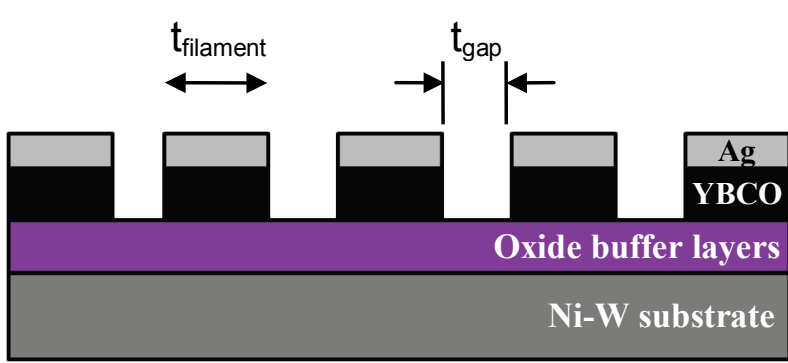

Fig. 1 Architecture of a multifilamentary YBCO film

Several different approaches have previously been used to fabricate the filamentary architectures described above; however, no standard method has been demonstrated that is applicable to low-cost manufacturing of practical conductors. There are many requirements for such a process, including the ability to precisely form the superconducting layer with interfilament spacing ideally less than 50 microns wide and to not damage the remaining active filaments or the substrate during the processing. Many approaches have been used to fabricate these low ac loss tapes including laser ablation [5-7], photolithography/chemical etching [8], mechanical scribing [2] and inkjet printing [9-10]. However, each method presents unique challenges in terms of its implementation into existing production processes. 


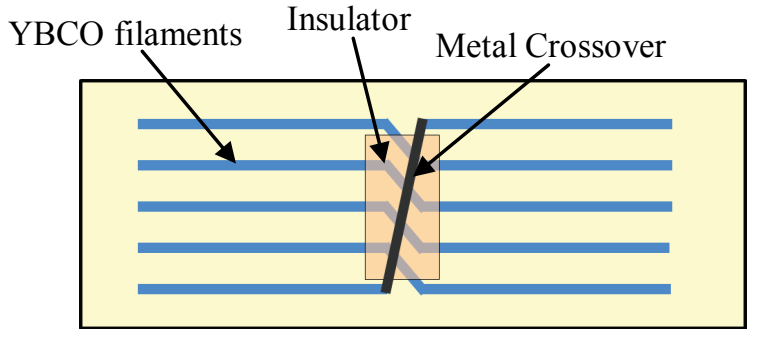

Fig. 2 Simulated twisted pair geometry for flat tape filamentary conductor

Here we propose an additive laser-based technique called laser direct-write to produce the YBCO superconductor filaments as well as the metallic cross-over bridges. Laser direct-write is a non-vacuum process that is performed in normal, ambient laboratory conditions. [11] It also has several other advantages that make it a unique solution for the fabrication of low ac loss structures using HTS tapes, including fast print speeds $(>1 \mathrm{~m} / \mathrm{sec})$ and manufacturability, in that it is viable as a scalable process to produce long lengths of tapes. In this paper, we describe the laser directwrite (LDW) process of metal-organic precursor solutions for YBCO filament formation and silver nanoinks for cross-over bridges. In addition, preliminary data will be presented revealing the viability of laser direct-write as a method to fabricate low ac loss HTS structures.

\section{Background}

Laser direct-write (LDW) refers to the laser-based modification, subtraction and addition of material without the use of masks or lithography. [12] It allows for the deposition of a variety of complex suspensions and inks, including metals, oxides and composites, directly onto virtually any substrate surface under ambient laboratory conditions. Because additive LDW is essentially a functional materials printer, it has proven successful in the fabrication of various components, including sensors, electronics and interconnects. [13-15] This process also offers design flexibility in that the desired pattern can be changed on-the-fly allowing for rapid prototyping. Since LDW is a non-contact process, it is well-suited for this application which requires the transfer of material onto flexible buffered substrates.

Recent technological advances by AMSC have allowed for the production of long-length superconducting tapes with the architecture seen in Figure 3. [16] Through the use of a metal organic decomposition (MOD) process, YBCO films can be made on these tapes. The MOD process is a non-vacuuum, solution-based technique in which the superconducting $\mathrm{YBCO}$ phase is produced from the decomposition and conversion of a trifluoroacetate (TFA) based precursor film. This process is used by AMSC to form the superconducting YBCO layer on their substrates fabricated on a reel-to-reel process via the RABiTS, (rolling-assisted biaxially textured susbtrates) method. [1719]

Our goal in this work is to use LDW to print the filamentized TFA-based precursor film onto buffered substrates, and to subsequently convert them to YBCO using a proprietary decomposition and reaction process. In addition, we use laser direct-write to print the metallic cross-over bridges needed to complete the simulated twisted pair geometry on the HTS flat tape architecture.

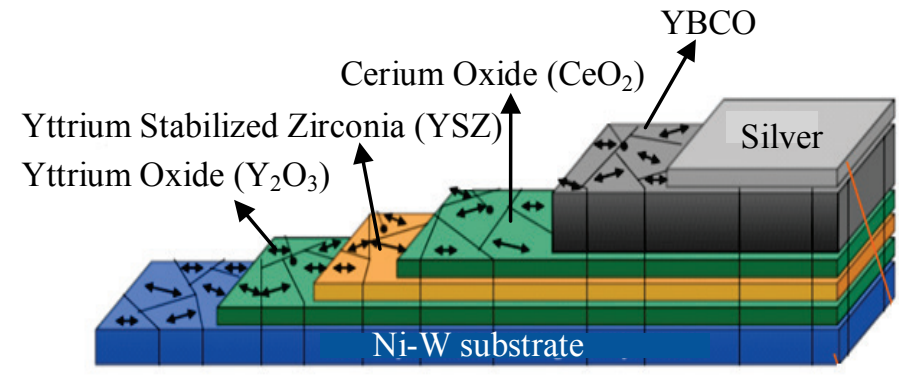

Fig. 3 Schematic of HTS tapes manufactured by AMSC

\section{Experimental Details}

TFA precursor solutions and RABiTS templates for the laser direct-write experiments were prepared by AMSC. The RABiTS templates consist of a Ni-W substrate with several buffer oxide layers $\left(\mathrm{Y}_{2} \mathrm{O}_{3} / \mathrm{YSZ} / \mathrm{CeO}_{2}\right)$ with a total thickness of $\sim 225 \mathrm{~nm}$. The standard precursor solution was modified by adding ethylene glycol to adjust the viscosity for laser printing. Spectrophotometry was performed to determine the optical absorption of the modified TFA precursor solution. As seen in Figure 4, absorption is highest at $\sim 260 \mathrm{~nm}$, making our 266nm UV laser ideallysuited for laser transfer.

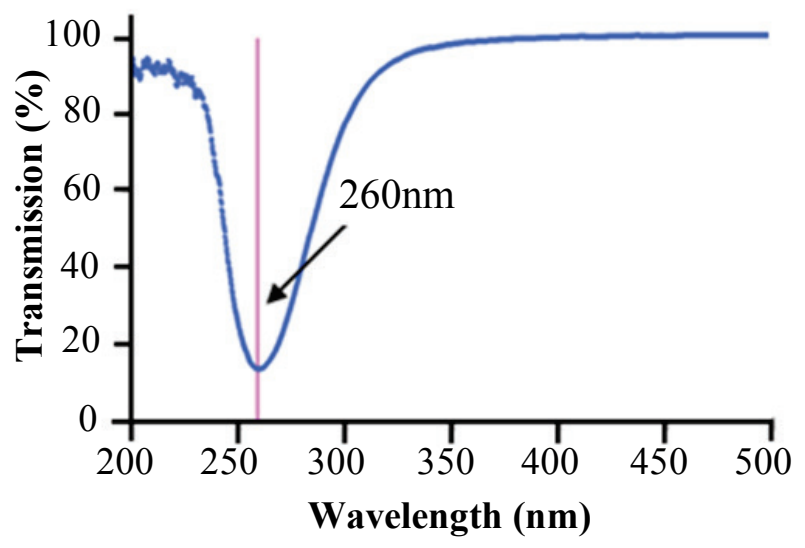

Fig. 4 Optical absorption spectrum for trifluoroacetatebased (TFA) precursor solution after ethylene glycol modification

The LDW transfers in this work were done using a frequency quadrupled $\mathrm{Nd}: \mathrm{YVO}_{4}$ laser operating at $266 \mathrm{~nm}$ at a repetition rate of $30 \mathrm{kHz}$. A schematic of the system is shown in Figure 5. An acousto-optic modulator (AOM) was used to control the amplitude of the laser pulses. Typical laser fluencies for transfers were between 5 and 50 $\mathrm{mJ} / \mathrm{cm}^{2}$. Two different methods were used to transfer the TFA precursor inks, depending on the resolution and overall scale needed. For narrow filaments, the laser pulses were imaged through an aperture and then a $10 \mathrm{X}, 0.25 \mathrm{NA}$ objective, forming a laser spot size of $\sim 50$ microns. The substrate was placed on top of motion-controlled X-Y translation stages. With this configuration, an in-line CCD 
camera was used to provide viewing of the transfer process. For wider filaments over a larger length scale (on the order of several centimeters), a galvanometer scanner was used to raster the laser beam, while the substrate remained stationary.

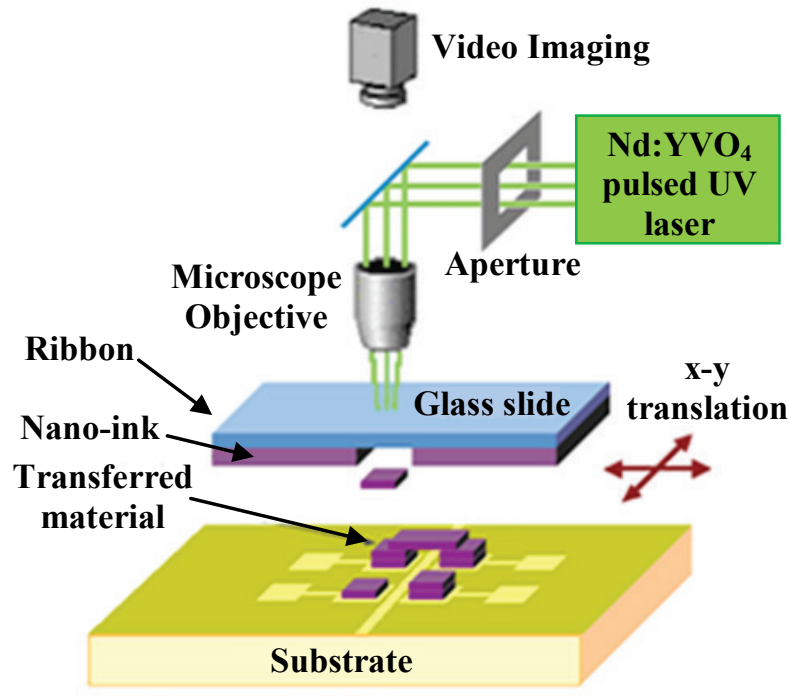

Fig. 5 Schematic of the laser direct-write system for the fabrication of narrow filaments

The transfer ribbon was made using a wire-coating technique, where a small amount of the TFA-based precursor solution was placed onto a 4" quartz wafer and then spread out onto the wafer using a metal wire-coater. The ribbon was then placed ink-side down on top of the receiving substrate, separated by an adjustable 12 to 50 micron gap. The laser spot was focused onto this ink layer and then pulsed to create lines by superimposing individual laser pulses, as the stages move.

Once the TFA precursor solutions were transferred to the receiving templates, they underwent a proprietary decomposition and conversion process, performed by AMSC. The laser transfers of the silver nano-ink were performed with an identical set-up and processing to that of the TFA-based precursors. The silver nano-ink used is commercially available and has a particle size of $\sim 10 \mathrm{~nm}$. These transfers were cured in an oven at $180^{\circ} \mathrm{C}$ for 45 minutes to fully sinter the silver to form a conductive pathway.

\section{Characterization of Laser Transfers}

An optical microscope was used to inspect the resulting transfers. To determine the width, thickness, and surface morphology of the transfers, a stylus profilometer (PLA Tencor P-16) was used. In addition, X-ray diffraction was used to determine YBCO crystal orientation and give insight into the quality of the film. Standard 4-point probe measurements were performed to determine the electrical quality of the printed silver nano-inks. The results of these characterization methods are discussed further in the following section.

\section{Discussion}

\subsection{Laser Direct-Write of TFA-based Precursor Filaments}

To verify that the LDW process is compatible with the materials system, transfers were performed using a 10x objective to print narrow lines. In Figure 6, these asprinted filaments can be seen on a silicon wafer and a glass substrate. The modified TFA precursors were successfully printed onto these substrates using LDW, showing proof of concept. The resulting material printed was several microns thick, as measured with a stylus profilometer, and was controlled by altering the number of laser passes used to print the lines.

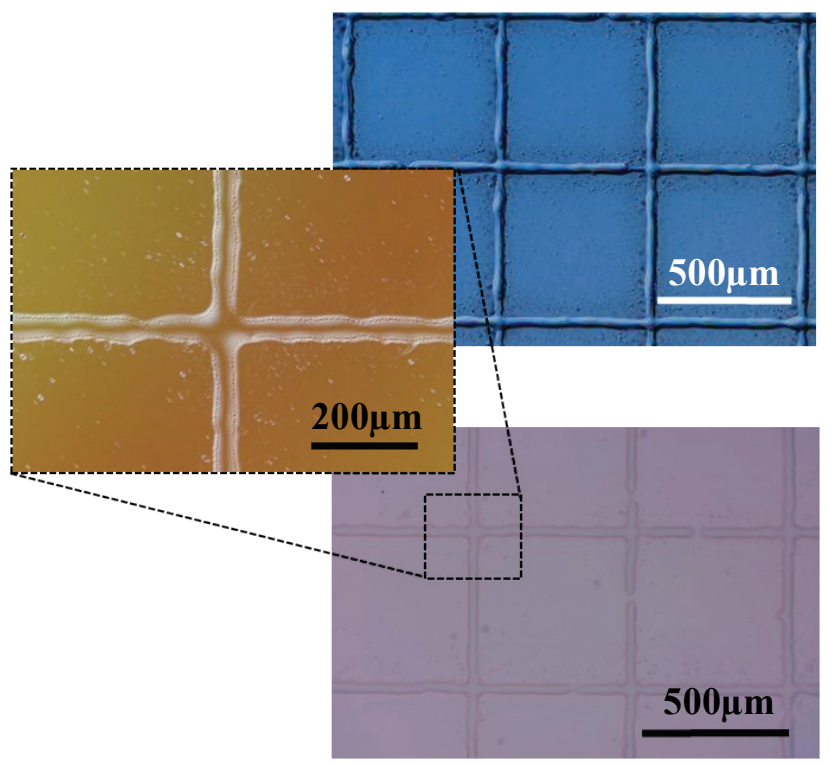

Fig. 6 As-printed TFA-based precursor lines onto a silicon wafer (top) and glass substrate (bottom)

An optical image of the TFA precursor transfers can be seen before and after decomposition in Figure 7. These samples were printed along the vertical direction using a galvanometer scanner, with the substrate remaining stationary. The $1 \mathrm{~mm}$ wide line was fabricated by printing several lines with an overlap of the laser spot to make a continuous structure.

After the decomposition, some striation was observed across the width of the sample. This is due to nonuniformity of the TFA-based precursor ink on the ribbon created by the wire-coating process. In addition, the decomposed structures exhibit some cracking, which is likely due to sub-optimal decomposition parameters for this system. The decomposition process is highly sensitive to temperature ramp rate and humidity levels and can affect the quality of the resulting YBCO film. In addition to cracking, the film shows signs of delamination along the vertical direction after decomposition, as seen in Figure 7. To achieve a laser transfer that resulted in a continuous conductive pathway, ethylene glycol was added to modify the precursor viscosity. It is possible that the addition of the ethylene glycol could affect the decomposition of the TFA precursors; however, a systematic optimization of the decomposition parameters was not carried out. 
a) Before decomposition (as-printed)
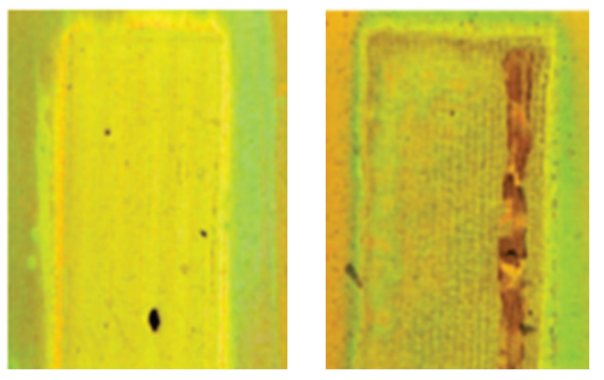

b) After decomposition (fully reacted)
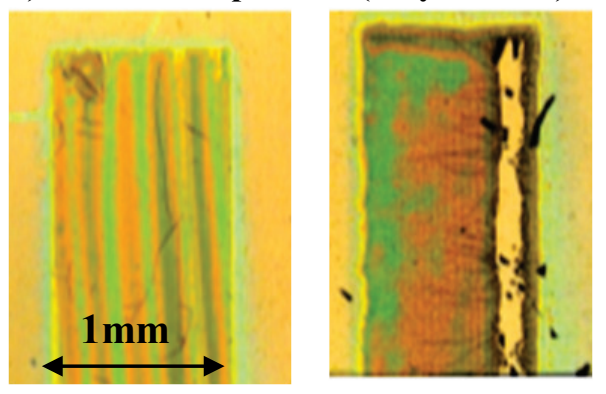

Fig. 7 TFA precursor filaments printed via LDW a) before decomposition and $b$ ) after decomposition, where striation can be seen on the left-side and delamination on the rightside

To verify that the decomposition of the laser transfers resulted in YBCO filaments, X-ray diffraction (XRD) was performed on fully reacted filaments. The 2- $\theta$ XRD data, seen in Figure 8, reveals that the filaments were successfully converted into c-axis textured YBCO. Because the pathway for conduction in YBCO occurs in the $\mathrm{Cu}_{2} \mathrm{O}$ planes, there exists transport anisotropy where conduction is approximately 10 times smaller along the $c$ axis then along the $\mathrm{Cu}_{2} \mathrm{O} a-b$ planes. This result is particularly important because it shows that YBCO can be grown in the preferential $c$-axis orientation onto a polycrystalline buffer layer via LDW. The YBCO filaments also exhibited a critical temperature of $85 \mathrm{~K}$. In addition, this confirms that the laser direct-write process is compatible with these materials, in that there is no damage to the TFA-based precursor solutions or the receiving buffer substrate.

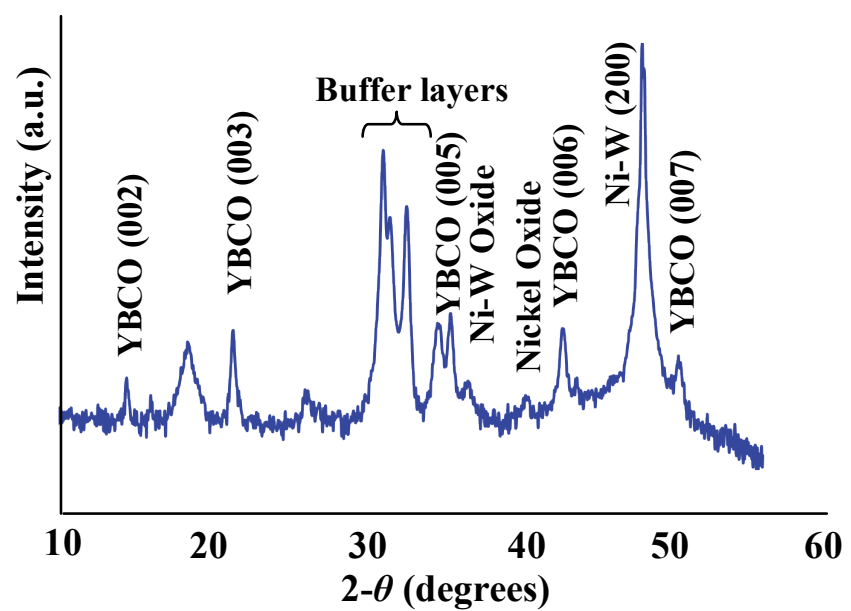

Fig. 8 X-ray diffraction data for a fully reacted YBCO filament printed via LDW
In addition, the LDW process is additive, so it does not require the removal of already grown $\mathrm{YBCO}$, resulting in less waste of material. With ablation and lithographic patterning techniques, the quality of the YBCO interface is compromised. By using an additive technique instead of subtractive, the integrity of the YBCO remains intact, with no damage to the surface or interface.

\subsection{Laser Direct-Write of Ag Cross-over Bridges}

It has been demonstrated that simply dividing the YBCO film into a filamented array does not aid in minimizing the ac loss alone, but rather requires the introduction of metallic cross-over bridges for flux penetration. [2, 20] These cross-over bridges cannot be fabricated via traditional lithographic manufacturing techniques and thus have proven to be a roadblock to the production of low ac loss HTS structures. It was demonstrated by Ashworth and Grilli, that to sufficiently decouple the YBCO filaments, the tapes require approximately five cross-over bridges per meter. [2] Laser direct-write provides a solution to this problem in that it is an additive, non-lithographic technique where Ag cross-over bridges can be added with relative ease along the filamented structure.

A commercially available silver nano-ink was transferred onto the YBCO surface of HTS tapes to form $1 \mathrm{~mm}$ wide lines, seen in Figure 9. The integrity of the silver lines is very important for this application because the contact resistance between the metallic cross-over bridges and the YBCO filaments must be low enough to aid in an overall reduction in ac losses. [2]

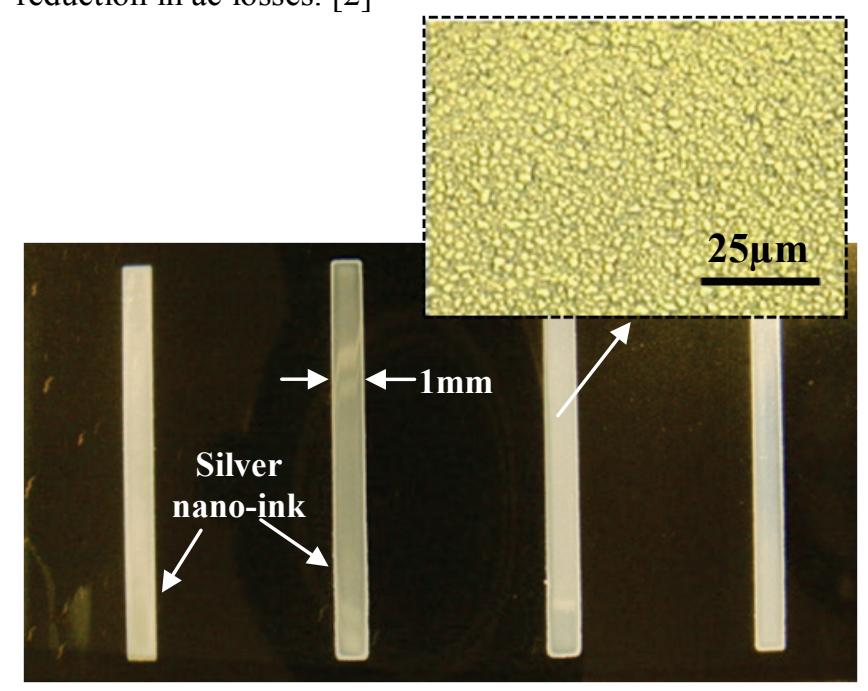

Fig. 9 Silver nanoink lines printed onto YBCO layer of HTS tape via LDW

To determine the quality of the Ag nano-ink laser printed lines, a Ag coating fabricated via vacuum deposition was used as a comparison for resistivity measurements. The data, seen in Figure 10, indicates the laser printed $\mathrm{Ag}$ makes an excellent, low resistance contact to the YBCO film that is comparable to that achieved with a vacuum deposited Ag layer, implying that the LDW Ag does not cause any measurable degradation of the underlying YBCO. This result is critical because it confirms that the LDW technique, along with the Ag nano- 
ink, is a viable method for the deposition of metallic crossover bridges.

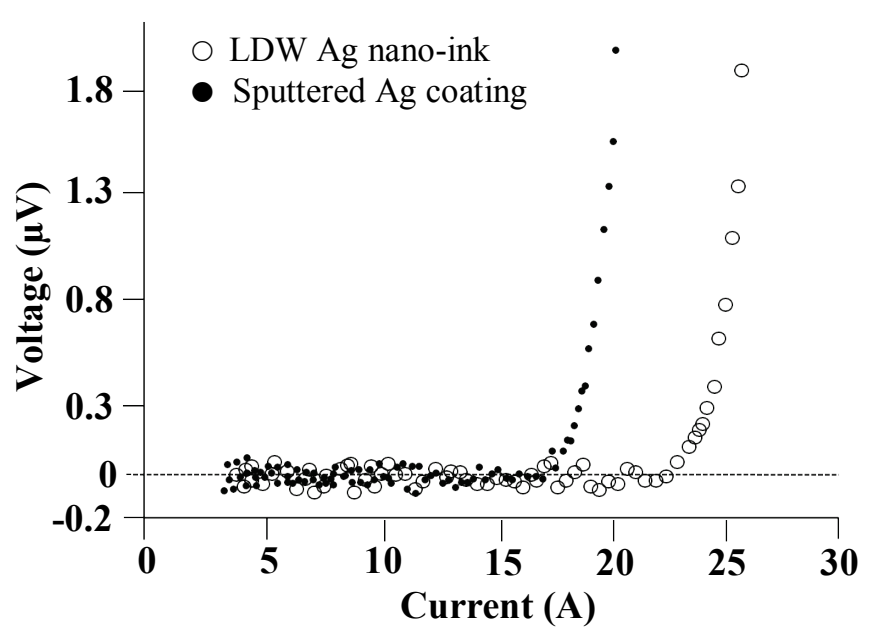

Fig. $10 \mathrm{I}-\mathrm{V}$ curves for Ag nano-ink lines printed via LDW (open circles) and sputtered Ag coating (closed circles)

\section{Summary}

This report describes the use of laser direct-write techniques for the fabrication of low ac loss high temperature superconductors. LDW was used to print TFA precursors filaments, which were then decomposed and reacted to form YBCO filaments on oxide buffered $\mathrm{Ni}-\mathrm{W}$ substrates. In addition, Ag nano-ink lines were printed onto HTS tapes for their use as cross-over bridges along the filament array. By using the LDW process to print both the TFA precursors and the Ag nano-ink lines, we are able to fabricate low ac loss HTS structures using one tool. Being able to manufacture the entire structure with one experimental setup is advantageous in that it simplifies the fabrication process significantly. These laser-based techniques have proven to be a promising methodology for the fabrication of low ac loss superconductors and could potentially be scalable for implementation into an existing HTS manufacturing environment.

\section{Acknowledgments}

The authors wish to acknowledge M. Rupich and S. Sathyamurthy at AMSC for providing materials and assisting in processing. This work was sponsored by the Office of Naval Research.

\section{References}

[1] X. Li, M.W. Rupich, C.L.H. Thieme, M. Teplitsky, S. Sathyamurthy, E. Thompson, E. Siegal, D. Buczek, J. Schreiber, K. DeMoranville, D. Hannus, J. Lynch, J. Inch, D. Tucker, R. Savoy, and S. Fleshler: IEEE Trans. Appl. Supercond., 19, 3, (2009) 3231-3235.

[2] S.P. Ashworth and F. Grilli: Supercond. Sci. Technol., 19, (2006) 227-232.

[3] E. Zeldov, J.R. Clem, M. McElfresh, and M. Darwin: Phys. Rev. B, 49, 14, (1994) 9802-9822.

[4] C.E. Oberly, G.L. Rhoads, P.N. Barnes, L. Long, D.J. Scott, and W.J. Carr: Adv. in Cryogenic Eng., 48, 614, (2002) 621-630.
[5] J. Souc, M. Vojenciak, F. Gömöry, X. Granados, A. Usoskin, and A. Rutt: Jour. of Phys.: Conf. Ser., 43, (2006) 127-129.

[6] Y. Zhang, R.C. Duckworth, T.T. Ha, F.A. List, III, M.J. Gouge, Y. Chen, X. Xiong, V. Selvamanickam, and A. Polyanskii: IEEE Trans. Appl. Supercond., 21, 3, (2011) 3301-3306.

[7] H. Okamoto, H. Hayashi, A. Tomioka, M. Konno, M. Owa, A. Kawagoe, F. Sumiyoshi, M. Iwakuma, K. Suzuki, T. Izumi, Y. Yamada, and Y. Shiohara: Physica C: Supercond., 468, 15-20, (2008) 1731-1733.

[8] D. Abraimov, A. Gurevich, A. Polyanskii, X.Y. Cai, A. $\mathrm{Xu}$, S. Pamidi, D. Larbalestier, and C.L.H. Thieme: Supercond. Sci. Technol., 21, (2008)

[9] F.A. List, T. Kodenkandath, and M.W. Rupich: IEEE Trans. Appl. Supercond., 17, 2, (2007) 3355-3358.

[10] R.C. Duckworth, M.P. Paranthaman, M.S. Bhuiyan, F.A. List, III, and M.J. Gouge: IEEE Trans. Appl. Supercond., 17, 2, (2007) 3159-3162.

[11]C.B. Arnold, P. Serra, and A. Piqué: MRS Bulletin, 32, (2007) 23-31

[12] J.W. Wang, R.C.Y. Auyeung, H. Kim, N.A. Charipar, and A. Piqué: Adv. Materials, 22, 40, (2010) 44624466.

[13]H. Kim, R.C.Y. Auyeung, and A. Piqué: Jour. of Power Sources, 165, 1, (2007) 413-419.

[14] M. Duocastella, H. Kim, P. Serra, and A. Piqué: Appl. Phys. A: Mat. Sci. and Proc., 106, 3, (2012) 471-478.

[15]H. Kim, R.C.Y. Auyeung, S.H. Lee, A.L. Huston, and A. Piqué: Jour. of Phys. D: Appl. Phys., 43, 8, (2010) 085101.

[16]A.P. Malozemoff and Y. Yamada: "100 Years of Superconductivity" ed. H. Rogalla and P. Kes, (CRC Press-Taylor \& Francis Group, 2012), pp.689-702.

[17]M.W. Rupich, D.T. Verebelyi, W. Zhang, T. Kodenkandath, and X. Li: MRS Bulletin, 29, (2004) 572.

[18]M.W. Rupich, X. Li, C. Thieme, S. Sathyamurthy, S. Fleshler, D. Tucker, E. Thompson, J. Schreiber, J. Lynch, D. Buczek, K. DeMoranville, J. Inch, P. Cedrone, and J. Slack: Supercond. Sci. Technol., 23, (2010) 014015, 1-9.

[19] M.W. Rupich, X. Li, S. Sathyamurthy, C. Thieme, and S. Fleshler: Physica C, 471, (2011) 919-923.

[20] W.T. Norris: J. Phys. D: Appl. Physics, 3, (1970) $489-$ 507.

(Received: June 30, 2012, Accepted: November 12, 2012) 